\title{
NUMERICALLY FLAT PRINCIPAL BUNDLES
}

\author{
INDRANIL BISWAS AND SWAMINATHAN SUBRAMANIAN
}

(Received May 12, 2003, revised July 29, 2004)

\begin{abstract}
Generalizing the notion of a numerically flat vector bundle over a Kähler manifold $M$, we define a numerically flat principal $G$-bundle over $M$, where $G$ is a semisimple complex algebraic group. It is proved that a principal $G$-bundle $E_{G}$ is numerically flat if and only if $\operatorname{ad}\left(E_{G}\right)$ is numerically flat. Numerically flat bundles are also characterized using the notion of semistability.
\end{abstract}

1. Introduction. Let $M$ be a compact Kähler manifold. In [DPS], the notion of a numerically effective holomorphic vector bundle over $M$ was introduced (see Section 2).

Let $G$ be a semisimple complex algebraic group. Let $P$ be a parabolic subgroup of $G$ and $\chi$ a character of $P$ anti-dominant with respect to some Borel subgroup of $G$ contained in $P$. So the line bundle over the projective variety $G / P$ defined by $\chi$ is numerically effective.

For a holomorphic $G$-bundle $E_{G}$ over $M$, the quotient map $E_{G} \rightarrow E_{G} / P$ defines a holomorphic principal $P$-bundle over $E_{G} / P$. The $G$-bundle $E_{G}$ will be called numerically flat if for all pairs $(P, \chi)$ the line bundle over $E_{G} / P$ defined by the anti-dominant character $\chi$ is numerically effective.

A principal SL $(n, \boldsymbol{C})$-bundle is numerically flat if and only if the vector bundle associated to it by the standard representation is numerically flat (Proposition 2.3). For a numerically flat $G$-bundle, any associated vector bundle is also numerically flat (Theorem 2.4). A $G$-bundle $E_{G}$ is numerically flat if and only if its adjoint vector bundle $\operatorname{ad}\left(E_{G}\right)$ is numerically flat (Theorem 2.5).

A numerically flat $G$-bundle $E_{G}$ is semistable and all the (rational) characteristic classes of $E_{G}$ of positive degree vanish. In the converse direction, if $M$ is a projective manifold and $E_{G}$ a semistable $G$-bundle over $M$ such that all the characteristic classes of $E_{G}$ of positive degree vanish, then $E_{G}$ is numerically flat (Theorem 3.1).

For a parabolic subgroup $P$ of $G$, its Levi quotient will be denoted by $L(P)$. For a principal $P$-bundle $E_{P}$, the principal $L(P)$-bundle obtained by extending the structure group using the projection of $P$ to $L(P)$ will be denoted by $E_{L(P)}$. A $G$-bundle $E_{G}$ over a Kähler manifold $M$ is numerically flat if and only if there is a parabolic subgroup $P \subset G$ and a reduction $E_{P} \subset E_{G}$ of structure group such that the principal $P$-bundle $E_{P}$ admits a flat holomorphic connection $\nabla$ with the property that the monodromy of the flat connection on $E_{L(P)}$ induced by $\nabla$ is contained in a maximal compact subgroup of $L(P)$ (Proposition 3.2).

2000 Mathematics Subject Classification. Primary 32L05; Secondary 53C05.

Key words and phrases. Principal bundle, numerically flat bundle, Kähler manifold. 
2. Numerically flatness. Let $M$ be a compact connected Kähler manifold equipped with a Kähler metric $\omega$. Let $L$ be a holomorphic line bundle over $M$. We recall from [DPS] the definition of a numerically effective line bundle.

DEFINITION 2.1 (DPS, Definition 1.2). The line bundle $L$ is called numerically effective if for any $\varepsilon>0$, there is a Hermitian metric $h_{\varepsilon}$ on it such that the curvature $\Theta_{h_{\varepsilon}}$ of the Chern connection on $L$ satisfies the inequality

$$
\Theta_{h_{\varepsilon}} \geq-\varepsilon \omega .
$$

Since $M$ is compact, the above definition clearly does not depend on the choice of $\omega$.

A vector bundle $E$ over $M$ is called numerically effective if the tautological line bundle $\mathcal{O}_{\boldsymbol{P}(E)}(1)$ over the projective bundle $\boldsymbol{P}(E)$ is numerically effective (cf. [DPS, p. 305, Definition 1.9]). A vector bundle $E$ over $M$ is called numerically flat if both $E$ and its dual $E^{*}$ are numerically effective (cf. [DPS, p. 311, Definition 1.17]).

Let $G$ be a semisimple linear algebraic group over the field $C$ of complex numbers. A Zariski closed proper subgroup $P$ of $G$ is called a parabolic subgroup if $G / P$ is compact.

Let $E_{G}$ be a holomorphic principal $G$-bundle over $M$. For a parabolic subgroup $P \subset G$, the projection $E_{G} \rightarrow E_{G} / P$ defines a holomorphic principal $P$-bundle. Given any character $\chi$ of $P$, let

$$
E_{G} \times{ }^{P} \boldsymbol{C}:=\left(E_{G} \times \boldsymbol{C}\right) / P
$$

be the associated line bundle over $E_{G} / P$; the quotient is for the action of $P$ defined as follows: the action of any $g \in P$ sends any point $(z, c) \in E_{G} \times C$ to $\left(z g, \chi\left(g^{-1}\right) c\right) \in E_{G} \times C$. This associated line bundle $E_{G} \times{ }^{P} C$ will be denoted by $L_{\chi}$.

DEFINITION 2.2. The $G$-bundle $E_{G}$ is called numerically flat if for every parabolic subgroup $P \subset G$ and every character $\chi: P \rightarrow C^{*}$ dominant with respect to some Borel subgroup of $G$ contained in $P$, the dual line bundle $L_{\chi}^{*}$ over $E_{G} / P$ is numerically effective in the sense of the above definition (Definition 2.1).

See $[\mathrm{Ra}]$ for the details on dominant characters of $P$. A character $\chi$ of $P$ is dominant if and only if the dual of the line bundle over $G / P$ associated to $\chi$ is numerically effective.

Since the pullback of a numerically effective line bundle is also numerically effective (see [DPS, p. 302, Proposition 1.8(i)]), and a line bundle $L$ is numerically effective if $L^{\otimes n}$ is numerically effective for some $n \geq 1$, it suffices to check the condition in Definition 2.2 only for maximal parabolic subgroups $P$. To explain this in more detail, for an arbitrary parabolic subgroup $Q$ of $G$ there are only finitely maximal parabolic subgroups $P_{i}$ of $G$ that contain $Q$. The ensuing map

$$
G / Q \rightarrow \prod_{Q \subset P_{i}} G / P_{i}
$$

is an embedding. Given any dominant character $\chi$ of $Q$, there are dominant characters $\chi_{i}$ of $P_{i}$ such that $\prod_{i} \chi_{i}$ on $Q$ coincides with $\chi$. Therefore, it is enough to check the condition in Definition 2.2 only for maximal parabolic subgroups. 
Let $E$ be a holomorphic vector bundle of rank $n$ over $M$ with $\wedge^{n} E \cong \mathcal{O}_{M}$. So $E$ defines a holomorphic principal $\operatorname{SL}(n, \boldsymbol{C})$-bundle over $M$. The principal $\operatorname{SL}(n, \boldsymbol{C})$-bundle defined by $E$ will be denoted by $E_{\mathrm{SL}}$.

PROPOSITION 2.3. The vector bundle $E$ is numerically flat if and only if the principal $\mathrm{SL}(n, \boldsymbol{C})$-bundle $E_{\mathrm{SL}}$ is numerically flat.

Proof. Assume that $E_{\mathrm{SL}}$ is numerically flat. Let $P \subset \mathrm{SL}(n, \boldsymbol{C})$ be the parabolic subgroup that fixes a given line $V_{0} \subset C^{n}$. Let $\chi$ be the character of $P$ defined by its action on $V_{0}$. So the quotient $E_{\mathrm{SL}} / P$ is $\boldsymbol{P}(E)$ and $\mathcal{O}_{\boldsymbol{P}(E)}(-1)$ is the line bundle associated to $\chi$. Therefore, from the definition of numerically flatness of $E_{\mathrm{SL}}$ we conclude that the vector bundle $E$ is numerically effective. Since $E$ is numerically effective and $\bigwedge^{n} E \cong \mathcal{O}_{M}$, we conclude that $E$ is numerically flat (see [DPS, p. 311, Definition 1.17], [DPS, p. 307, Proposition 1.14(iii)]).

Now assume that $E$ is numerically flat. Maximal parabolic subgroups of $\operatorname{SL}(n, C)$ are those that preserve some subspace of $\boldsymbol{C}^{n}$. The quotient is a Grassmannian. Let $\operatorname{Gr}(E, k)$ be the Grassmann bundle over $M$ consisting of all $k$ dimensional subspaces in the fibers of $E$, where $k \in[1, n-1]$. The condition that $E$ is numerically effective implies that the vector bundle $\wedge^{k} E$ is also numerically effective [DPS, p. 307, Proposition 1.14(ii)]. The line bundles over $\operatorname{Gr}(E, k)$ corresponding to the dominant characters are the nonnegative powers of the line bundle over $\operatorname{Gr}(E, k)$ defined by the determinant of the tautological vector bundle of rank $k$. Since the determinant of the rank $k$ tautological vector bundle over $\operatorname{Gr}(E, k)$ is the pullback of $\mathcal{O}_{P\left(\wedge^{k} E\right)}(-1)$ using the Plücker embedding, the numerically effectiveness of $\wedge^{k} E$ implies that the dual of the determinant of the tautological vector bundle over $\operatorname{Gr}(E, k)$ is numerically effective.

Proposition 2.3 justifies Definition 2.2.

Let $V$ be a finite-dimensional complex $G$-module. For any $G$-bundle $E_{G}$, the quotient $E_{G} \times{ }^{G} V:=\left(E_{G} \times V\right) / G$ for the twisted diagonal action is a vector bundle, which is called the associated vector bundle.

The following theorem, which is proved using a basic result due to C. Mourougane, is similar in spirit to the characterization of semistable $G$-bundles in terms of the semistability of the associated vector bundles (see [RS, Theorem 3], [AB, Proposition 2.10]).

THEOREM 2.4. Let $E_{G}$ be a numerically flat $G$-bundle over $M$. For any finite dimensional complex $G$-module $V$ the associated vector bundle $E_{G} \times{ }^{G} V$ is numerically flat.

Proof. Since $V$ is a direct sum of irreducible $G$-modules, and a direct sum of numerically flat vector bundles is again numerically flat, it suffices to prove the theorem for irreducible $G$-modules. So assume $V$ to be irreducible.

From the Borel-Weil-Bott theorem we know that there is a parabolic subgroup $P$ of $G$ and an anti-dominant character (inverse of a dominant character) $\chi$ of $P$ such that the associated line bundle $\mathcal{L}_{\chi}=G \times{ }^{P} \boldsymbol{C}$ over $G / P$ is ample, and the induced representation of $G$ on $H^{0}\left(G / P, L_{\chi}\right)$ coincides with the $G$-module $V$ (cf. [Bo]). 
Let $p: E_{G} / P \rightarrow M$ be the natural projection. The above form of the Borel-Weil-Bott theorem immediately implies that the associated line bundle $L_{\chi}=E_{G} \times{ }^{P} \boldsymbol{C}$ over $E_{G} / P$ has the property that

$$
p_{*} L_{\chi} \cong E_{G} \times{ }^{G} V
$$

Let $K_{\text {rel }}^{-1}:=K_{E_{G} / P}^{-1} \otimes p^{*} K_{M}$ be the relative anti-canonical line bundle over $E_{G} / P$. Note that the anti-canonical bundle $K_{G / P}^{-1}$ over $G / P$ corresponds to an anti-dominant character (as $K_{G / P}^{-1}$ is ample) $\hat{\chi}$ of $P$, that is, $K_{G / P}^{-1}$ is the line bundle associated to $\hat{\chi}$. So the character $\chi \hat{\chi}$ of $P$ is anti-dominant.

The line bundle $L_{\chi \hat{\chi}}=E_{G} \times{ }^{P} \boldsymbol{C}$ over $E_{G} / P$ associated to the character $\chi \hat{\chi}$ is clearly $L_{\chi} \otimes K_{\text {rel }}^{-1}$. If $E_{G}$ is numerically flat, we know from the definition that $L_{\chi} \otimes K_{\text {rel }}^{-1}$ is numerically effective. Since the restriction of $L_{\chi} \otimes K_{\text {rel }}^{-1}$ to any fiber of the projection $p$ is an ample line bundle, the direct image

$$
p_{*}\left(K_{\mathrm{rel}} \otimes L_{\chi} \otimes K_{\mathrm{rel}}^{-1}\right) \cong p_{*} L_{\chi}
$$

is numerically effective (cf. [Mo, p. 895, Théorème 2]). This theorem of [Mo] says that if $L$ is a numerically effective line bundle over $E_{G} / P$ whose restriction to any fiber of $p$ is ample, then the direct image $p_{*}\left(K_{\mathrm{rel}} \otimes L\right)$ is a numerically effective vector bundle over $M$. The above assertion is obtained by setting $L=L_{\chi} \otimes K_{\text {rel }}^{-1}$.

Finally using the isomorphism in (2.1) we conclude that the associated vector bundle $E_{G} \times{ }^{G} V$ is numerically effective if $E_{G}$ is numerically flat. Since $G$ does not have any nontrivial character (as it is semisimple) we have $\bigwedge^{\text {top }} E_{G} \times{ }^{G} V \cong \mathcal{O}_{M}$. Therefore, it follows that $\operatorname{ad}\left(E_{G}\right)$ is numerically flat if $E_{G}$ is so.

Let $\mathfrak{g}$ denote the Lie algebra of $G$, on which $G$ acts by conjugation. Since $G$ is semisimple, the kernel of the homomorphism

$$
\rho: G \rightarrow \operatorname{SL}(\mathfrak{g})
$$

is a finite group. For a principal $G$-bundle $E_{G}$, the associated adjoint bundle $E_{G} \times{ }^{G} \mathfrak{g}$ will be denoted by $\operatorname{ad}\left(E_{G}\right)$.

For a parabolic subgroup $P$ of $G$, let $R_{u}(P)$ denote the unipotent radical of $P$. So $R_{u}(P)$ is the (unique) maximal connected unipotent normal subgroup of $P$. The quotient $L(P):=P / R_{u}(P)$ is called the Levi factor of $P$. The group $L(P)$ is reductive. (See [Bor].)

THEOREM 2.5. Let $V$ be as in Theorem 2.4 such that the kernel of the homomorphism $G \rightarrow \mathrm{SL}(V)$ is a finite group. A G-bundle $E_{G}$ is numerically flat if and only if the associated vector bundle $E_{G} \times{ }^{G} V$ is numerically flat. In particular, $E_{G}$ is numerically flat if and only if $\operatorname{ad}\left(E_{G}\right)$ is numerically flat.

Proof. If $E_{G}$ is numerically flat, then Theorem 2.4 implies that the vector bundle $E_{G} \times{ }^{G} V$ is numerically flat. 
Let $E_{\mathrm{SL}(V)}$ be the principal SL(V)-bundle over $M$ obtained by extending the structure group of $E_{G}$ using the homomorphism

$$
\tau: G \rightarrow \operatorname{SL}(V)
$$

defined by the action of $G$ on $V$.

For any maximal parabolic subgroup $P$ of $G$ and any dominant character $\chi$ of $P$, there is a parabolic subgroup $Q$ (not necessarily maximal) of $\operatorname{SL}(V)$ and a dominant character $\chi^{\prime}$ of $Q$ such that $\tau(P)=\tau(G) \cap Q$ and $\tau^{*} \chi^{\prime}=\chi^{n}$ for some $n \geq 1$. To prove this first note that since $P$ is maximal parabolic the group of characters of $P$ is isomorphic to $Z$. If $\tau(P)=\tau(G) \cap Q$, then $G / P$ embeds into $\operatorname{SL}(V) / Q$. Since the pullback of a numerically effective line bundle is numerically effective, we have $\tau^{*} \chi^{\prime}=\chi^{n}$ for some $n \geq 1$. So all we need to show is that there is a parabolic subgroup $Q$ with $\tau(G) \cap Q=\tau(P)$.

Let $N_{1} \subset \mathrm{SL}(V)$ be the normalizer of the subgroup $\tau\left(R_{u}(P)\right)$, where $\tau$ is defined in (2.3). Let $R_{1} \subset N_{1}$ be its unipotent radical. Inductively define $N_{k+1}$ to be the normalizer of $R_{k}$, and $R_{k+1}$ to be the unipotent radical of $N_{k+1}$. Both $\left\{N_{i}\right\}_{i \geq 1}$ and $\left\{R_{i}\right\}_{i \geq 1}$ are increasing subgroups of $\operatorname{SL}(V)$. Note that each $N_{i}$ is a proper subgroup of $\operatorname{SL}(V)$, as it is a normalizer of a nontrivial unipotent subgroup (the unipotent subgroup is nontrivial as the kernel of the homomorphism $G \rightarrow \operatorname{SL}(V)$ is finite). The limiting group, call it $Q$, of $\left\{N_{i}\right\}_{i \geq 1}$ has the property that the normalizer of the unipotent radical of $Q$ is $Q$ itself. This implies that $Q$ is a parabolic subgroup of $\operatorname{SL}(V)$. (The assumption that the kernel of the homomorphism $G \rightarrow \operatorname{SL}(V)$ is finite ensures that $Q$ is a proper subgroup of $\operatorname{SL}(V)$.) The parabolic group $Q$ clearly has the property that $Q \cap \tau(G)=\tau(P)$.

Consequently, we have an embedding of $E_{G} / P$ in $E_{\mathrm{SL}(V)} / Q$, and the line bundle over $E_{G} / P$ defined by $\chi^{n}$ coincides with the restriction of the line bundle over $E_{\mathrm{SL}(V)} / Q$ defined by $\chi^{\prime}$. Therefore, $E_{G}$ is numerically flat if $E_{\mathrm{SL}(V)}$ is so.

REMARK 2.6. Let

$$
\sigma: G \rightarrow H
$$

be a homomorphism to a complex semisimple group $H$. Using $\sigma$ the Lie algebra $\mathfrak{h}$ of $H$ is a left $G$-module. Consider the principal $H$-bundle $E_{H}:=E_{G} \times{ }^{G} H$ obtained by extending the structure group of $E_{G}$ using $\sigma$. Since that adjoint vector bundle $\operatorname{ad}\left(E_{H}\right)$ is the one associated to $E_{G}$ for the $G$-module $\mathfrak{h}$, if $E_{G}$ is numerically flat then Theorem 2.4 and Theorem 2.5 combine together to imply that $E_{H}$ is numerically flat.

3. Semistability and numerical flatness. Let $F$ be a holomorphic vector bundle defined on a dense open subset $U \subset M$ such that the complement $M \backslash U$ is a complex analytic subset of (complex) codimension at least two. Let $\iota: U \hookrightarrow M$ be the inclusion map. The condition on the codimension of $M \backslash U$ implies that the direct image $\iota_{*} F$ is a coherent sheaf on $M$. The degree of $F$ is defined as 


$$
\operatorname{deg}(F):=\int_{M} c_{1}\left(\iota_{*} F\right) \omega^{d-1},
$$

where $d=\operatorname{dim} M$ and $\omega$ is the fixed Kähler form on $M$.

A principal $G$-bundle $E_{G}$ over $M$ is called semistable (respectively, stable) if for any reduction of structure group $\left.E_{P} \subset E_{G}\right|_{U}$ to any parabolic subgroup $P$ over an open subset $U$, with $\operatorname{codim}(M \backslash U) \geq 2$, and any nontrivial character $\chi$ of $P$ dominant with respect to some Borel subgroup contained in $P$, the associated line bundle $L_{\chi}=E_{P} \times{ }^{P} C$ over $U$ satisfies the condition

$$
\operatorname{deg}\left(L_{\chi}\right) \leq 0
$$

(respectively, $\left.\operatorname{deg}\left(L_{\chi}\right)<0\right)$ (see [Ra], [RS], [AB]).

Take $P$ to be a maximal parabolic subgroup in the above definition. Let $\iota$ be the inclusion map of $U$ in $M$ and $\sigma: U \rightarrow E_{G} / P$ the section of the projection $E_{G} / P \rightarrow M$ defining the reduction of structure group to $P$. The above the inequality can be replaced by the inequality

$$
\operatorname{deg}\left(\iota_{*} \sigma^{*} T_{\mathrm{rel}}\right) \geq 0
$$

(respectively, $\operatorname{deg}\left(\iota_{*} \sigma^{*} T_{\text {rel }}\right)>0$ ), where $T_{\text {rel }}$ is the relative tangent bundle for the projection $E_{G} / P \rightarrow M$; see [Ra, Lemma 2.1] for a proof that the two formulations of the definition of (semi)stability are equivalent.

THEOREM 3.1. Let $E_{G}$ be a principal $G$-bundle over a Kähler manifold $M$. If $E_{G}$ is numerically flat, then $E_{G}$ is semistable and all the (rational) characteristic classes of $E_{G}$ of degree at least one vanish.

If $E_{G}$ is semistable and all the (rational) characteristic classes of $E_{G}$ of degree at least one vanish, then $E_{G}$ is numerically flat provided $M$ is a projective manifold.

Proof. Let $E_{G}$ be a numerically flat $G$-bundle over $M$. Theorem 2.5 says that the adjoint vector bundle $\operatorname{ad}\left(E_{G}\right)$ is numerically flat. From [DPS, p. 311, Theorem 1.18] it follows that $\operatorname{ad}\left(E_{G}\right)$ is semistable. The semistability of $\operatorname{ad}\left(E_{G}\right)$ implies that the $G$-bundle $E_{G}$ is semistable (cf. [AB, Proposition 2.10]).

Writing $G$ as a product of simple groups we see that it is enough to prove that all the higher characteristic classes (higher than degree zero) of $E_{G}$ vanish assuming that $G$ is simple. But for $G$ simple, all the characteristic classes of $E_{G}$ are contained in the characteristic classes of the adjoint vector bundle $\operatorname{ad}\left(E_{G}\right)$. As $\operatorname{ad}\left(E_{G}\right)$ is numerically flat, all the higher Chern classes (higher than degree zero) of $\operatorname{ad}\left(E_{G}\right)$ vanish (cf. [DPS, p. 311, Corollary 1.19]). Consequently, all the characteristic classes of $E_{G}$ of positive degree vanish.

Now assume that $M$ is a projective manifold and $E_{G}$ a semistable principal $G$-bundle over $M$ such that all the characteristic classes of $E_{G}$ of positive degree vanish.

The semistability of $E_{G}$ implies that the vector bundle $\operatorname{ad}\left(E_{G}\right)$ is semistable (cf. [RS, Theorem 3], [AB, Proposition 2.10]). Since all the characteristic classes of $E_{G}$ of positive degree vanish, it follows immediately that $c_{i}\left(\operatorname{ad}\left(E_{G}\right)\right)=0$ for all $i \geq 1$. 
Since $\operatorname{ad}\left(E_{G}\right)$ is semistable with vanishing Chern classes, Theorem 2 of [Si, p. 39] says that there is a filtration

$$
0=F_{0} \subset F_{1} \subset F_{2} \subset \cdots \subset F_{k-1} \subset F_{k}=\operatorname{ad}\left(E_{G}\right)
$$

of $\operatorname{ad}\left(E_{G}\right)$ by holomorphic subbundles such that each quotient vector bundle $F_{i} / F_{i-1}, i \in$ $[1, k]$, is a stable vector bundles with $c_{j}\left(F_{i} / F_{i-1}\right)=0$ for all $j \geq 1$. To deduce this from [Si, p. 39, Theorem 2] simply set the Higgs field to be zero in [Si, Theorem 2]; we need the assumption that $M$ is projective to be able to use this result of Simpson.

Now a theorem due to Donaldson [Do] and Uhlenbeck-Yau [UY] says that $F_{i} / F_{i-1}$ admits a unitary flat connection. Consequently, the vector bundle $F_{i} / F_{i-1}$ is numerically flat by [DPS, p. 311, Theorem 1.18]. Since an extension of a numerically flat vector bundle by a numerically flat vector bundle is again numerically flat by [DPS, p. 308, Proposition 1.15(ii)], using (3.1) it follows immediately that $\operatorname{ad}\left(E_{G}\right)$ is numerically flat. Now Theorem 2.5 says that the $G$-bundle $E_{G}$ is numerically flat.

Let $E_{P}$ be a holomorphic principal $P$-bundle over the compact Kähler manifold $M$ equipped with a holomorphic flat connection $\nabla$, where $P$ is a parabolic subgroup of $G$. Let $E_{L(P)}:=E_{P} \times{ }^{P} L(P)$ be the corresponding principal $L(P)$-bundle, where $L(P)$ is the Levi factor (defined prior to Theorem 2.5); here $P$ acts on $L(P)$ on the left using the projection of $P$ to $L(P)$. The connection $\nabla$ on $E_{P}$ induces a connection $\nabla^{L(P)}$ on $E_{L(P)}$, which is flat holomorphic as $\nabla$ is so.

PROPOSITION 3.2. A holomorphic $G$-bundle $E_{G}$ over a compact Kähler manifold $M$ is numerically flat if and only if there is a parabolic subgroup $P \subset G$, a reduction of structure group $E_{P} \subset E_{G}$, and a flat holomorphic connection $\nabla$ on the $P$-bundle $E_{P}$ such that the monodromy of the connection $\nabla^{L(P)}$ on $E_{L(P)}$ is contained in some maximal compact subgroup of $L(P)$.

Proof. Let $E_{P} \subset E_{G}$ be a reduction of structure group to a parabolic subgroup $P$ and $\nabla$ a flat holomorphic connection on $E_{P}$ with the above property. There is a parabolic subgroup $Q \subset \operatorname{SL}(\mathfrak{g})$, where $\mathfrak{g}$ is the Lie algebra of $G$, such that for the adjoint representation $\rho$ (defined in (2.2)) we have $\rho(P)=\rho(G) \cap Q$. The construction of $Q$ is given in the proof of Theorem 2.5.

Let $E_{Q}=E_{P} \times{ }^{P} Q$ be the principal $Q$-bundle obtained by extending the structure group of $E_{P}$ using $\rho$. Note that the adjoint vector bundle ad $\left(E_{G}\right)$ is the vector bundle associated (by the standard action) to the $\mathrm{SL}(\mathfrak{g})$-bundle obtained by extending the structure group of $E_{G}$ using $\rho$. The $Q$-bundle $E_{Q}$ is a reduction of structure group to $Q$ of $\operatorname{ad}\left(E_{G}\right)$, as $E_{Q}$ is the extension of structure group of $E_{P}$ using $\rho$. So the reduction $E_{Q}$ defines a filtration

$$
0=V_{0} \subset V_{1} \subset V_{2} \subset \cdots \subset V_{k-1} \subset V_{k}=\operatorname{ad}\left(E_{G}\right)
$$

of the vector bundle $\operatorname{ad}\left(E_{G}\right)$ by holomorphic subbundles.

Let $\nabla^{Q}$ denote the flat holomorphic connection on $E_{Q}$ defined by the connection $\nabla$ on $E_{P}$. So $\nabla^{Q}$ preserves the filtration in (3.2). Consequently, $\nabla^{Q}$ induces a flat holomorphic 
connection on each subsequent quotient $V_{i} / V_{i-1}$ in (3.2). The corresponding connection on the graded vector bundle $\bigoplus_{i=1}^{k} V_{i} / V_{i-1}$ coincides with the one induced by the connection $\nabla^{L(P)}$ on $E_{L(P)}$. Indeed, the homomorphism $\rho: P \rightarrow Q$ (in (2.2)) induces a homomorphism $L(P) \rightarrow L(Q)$ of the Levi quotients. Using this induced homomorphism the $L(P)$-bundle $E_{L(P)}$ gives a $L(Q)$-bundle $E_{L(Q)}$ (by extension of structure group). The vector bundle associated to $E_{L(Q)}$ for the action of $L(Q)$ on the graded vector space (for the filtration of $\mathfrak{g}$ that $Q$ preserves) is identified with $\bigoplus_{i=1}^{k} V_{i} / V_{i-1}$. So a connection on $E_{L(P)}$ gives a connection on $\bigoplus_{i=1}^{k} V_{i} / V_{i-1}$ by inducing a connection on $E_{L(Q)}$. Clearly, the connection on $\bigoplus_{i=1}^{k} V_{i} / V_{i-1}$ obtained from $\nabla^{Q}$ (constructed as above) coincides with the one given by $\nabla^{L(P)}$.

Since the monodromy of the flat connection $\nabla^{L(P)}$ is contained in some maximal compact subgroup of $L(P)$ it follows immediately that the connection on $V_{i} / V_{i-1}$ preserves some Hermitian structure on $V_{i} / V_{i-1}$. In other words, each $V_{i} / V_{i-1}$ admits a unitary flat connection. This implies that $\operatorname{ad}\left(E_{G}\right)$ is numerically flat by [DPS, p. 311, Theorem 1.18]. Consequently, by Theorem 2.5 the $G$-bundle $E_{G}$ is numerically flat.

To prove the converse, let $E_{G}$ be a numerically flat $G$-bundle over $M$. So $\operatorname{ad}\left(E_{G}\right)$ is numerically flat (Theorem 2.5). Now [DPS, p. 311, Theorem 1.18] says that $\operatorname{ad}\left(E_{G}\right)$ admits a filtration by holomorphic subbundles such that each successive quotient is a stable vector bundle with vanishing Chern classes of every positive degree.

Let

$$
0=W_{0} \subset W_{1} \subset W_{2} \subset \cdots \subset W_{k-1} \subset W_{k}=\operatorname{ad}\left(E_{G}\right)
$$

be the filtration of the vector bundle ad $\left(E_{G}\right)$ defined by socle. In other words, $W_{i} / W_{i-1}$ is the (unique) maximal polystable subsheaf of degree zero (the socle) of $\operatorname{ad}\left(E_{G}\right) / W_{i-1}$ (see $[\mathrm{AB}$, p. 211, Lemma 2.5] for properties of the socle).

Since $\operatorname{ad}\left(E_{G}\right)$ admits a filtration by holomorphic subbundles such that each successive quotient is a stable vector bundle with vanishing higher Chern classes, it follows immediately that each subsheaf $W_{i}$ in (3.3) is a subbundle of $\operatorname{ad}\left(E_{G}\right)$. Furthermore, for the same reason $c_{j}\left(W_{i} / W_{i-1}\right)=0$ for all $i, j \geq 1$. (If $F$ is a subbundle of degree zero of a polystable vector bundle $E$ with $c_{j}(E)=0$ for all $j \geq 1$, then $c_{j}(F)=0=c_{j}(E / F)$ for all $j \geq 1$.)

From the above properties of $\operatorname{ad}\left(E_{G}\right)$ it follows that it has a natural flat holomorphic connection $\nabla$ that preserves the filtration in (3.3), and the connection on each successive quotient $W_{i} / W_{i-1}$ induced by $\nabla$ is unitary flat (cf. [Si, p. 40, Corollary 3.10]). The construction of the connection $\nabla$ in [Si] needs the base manifold to be projective. But this assumption is only needed to conclude that the vector bundle admits a filtration by subbundles such that each successive quotient is stable of degree zero, that is, to have [ $\mathrm{Si}, \mathrm{p} .39$, Theorem 2] valid for the vector bundle. But using the assumption that $\operatorname{ad}\left(E_{G}\right)$ is numerically flat we already have such a filtration in (3.3).

Let $\mathcal{V} \subset \operatorname{End}\left(\operatorname{ad}\left(E_{G}\right)\right)$ be the subbundle that preserves the filtration in (3.3). So for any point $x \in M$ and any endomorphism $T \in \operatorname{End}\left(\operatorname{ad}\left(E_{G}\right)_{x}\right)$ we have $T \in \mathcal{V}_{x}$ if and only if $T\left(\left(W_{i}\right)_{x}\right) \subset\left(W_{i}\right)_{x}$ for all $i \geq 1$. The Lie algebra structure of the fibers of $\operatorname{ad}\left(E_{G}\right)$ define a 
homomorphism of vector bundles

$$
\tau: \operatorname{ad}\left(E_{G}\right) \rightarrow \operatorname{End}\left(\operatorname{ad}\left(E_{G}\right)\right) .
$$

Since $G$ is semisimple, its Lie algebra $\mathfrak{g}$ has trivial center. Hence the above homomorphism $\tau$ is pointwise injective.

Consider the intersection $\mathcal{V} \cap \tau\left(\operatorname{ad}\left(E_{G}\right)\right)$ inside $\operatorname{End}\left(\operatorname{ad}\left(E_{G}\right)\right)$. For each $x \in M$, this intersection defines a parabolic subalgebra of $\operatorname{ad}\left(E_{G}\right)_{x}$. Since the normalizer (inside $G$ ) of a parabolic subgroup $P \subset G$ is $P$ itself, the subalgebra bundle $\mathcal{V} \cap \tau\left(\operatorname{ad}\left(E_{G}\right)\right)$ defines a reduction of structure group $E_{P} \subset E_{G}$ to a parabolic subgroup $P$ such that $\operatorname{ad}\left(E_{P}\right)=\mathcal{V} \cap$ $\tau\left(\operatorname{ad}\left(E_{G}\right)\right)$ (see the construction of this reduction in the last paragraph of p. 341 in [ABi]).

Using the Killing form on $\mathfrak{g}$, the vector bundle $\operatorname{ad}\left(E_{G}\right)$ gets identified with $\operatorname{ad}\left(E_{G}\right)^{*}$. Indeed, since the Killing form is $G$-invariant, it defines a nondegenerate symmetric bilinear form on $\operatorname{ad}\left(E_{G}\right)$. Consider the vector bundle $\mathcal{W}:=\bigwedge^{2} \operatorname{ad}\left(E_{G}\right)$. The Lie algebra structure on the fibers of $\operatorname{ad}\left(E_{G}\right)$ gives a nowhere vanishing section

$$
s \in H^{0}(M, \mathcal{W})
$$

using the identification of $\operatorname{ad}\left(E_{G}\right)$ with its dual. Let $L_{s} \subset \mathcal{W}$ be the trivial line subbundle generated by $s$.

The canonical flat connection $\nabla$ on $\operatorname{ad}\left(E_{G}\right)$ constructed in [Si] induces a flat connection $\widetilde{\nabla}$ on $\mathcal{W}$. Since $L_{S}$ is a trivial line subbundle of $\mathcal{W}$, the connection $\widetilde{\nabla}$ preserves $L_{s}$, and the induced connection on $L_{s}$ is the trivial connection, that is, it has trivial monodromy; this property of $\widetilde{\nabla}$ follows immediately from the general properties of the connection constructed in $[\mathrm{Si}]$.

Consequently, the connection $\nabla$ on $\operatorname{ad}\left(E_{G}\right)$ is compatible with the Lie algebra structure of the fibers. The following lemma shows that the connection $\nabla$ induces a connection on the $G$-bundle $E_{G}$.

LEMMA 3.3. Let $E_{G}^{\prime}$ be a smooth principal $G$-bundle over a smooth manifold $M^{\prime}$, where $G$ is a semisimple linear algebraic group defined over $C$. Let $\nabla^{\prime}$ be a connection on the adjoint vector bundle $\operatorname{ad}\left(E_{G}^{\prime}\right)$ such that

$$
\left[\nabla_{v}^{\prime}(s), t\right]+\left[s, \nabla_{v}^{\prime}(t)\right]=\nabla_{v}^{\prime}([s, t])
$$

for all locally defined sections $s, t$ of $\operatorname{ad}\left(E_{G}^{\prime}\right)$ and all locally defined vector field on $M^{\prime}$. (The Lie algebra structure of the fibers of $\operatorname{ad}\left(E_{G}^{\prime}\right)$ gives a smooth section of the vector bundle $\operatorname{ad}\left(E_{G}^{\prime}\right) \otimes \operatorname{ad}\left(E_{G}^{\prime}\right)^{*} \otimes \operatorname{ad}\left(E_{G}^{\prime}\right)^{*}$; the above condition on $\nabla^{\prime}$ is equivalent to the condition that this section is flat with respect to the connection on $\operatorname{ad}\left(E_{G}^{\prime}\right) \otimes \operatorname{ad}\left(E_{G}^{\prime}\right)^{*} \otimes \operatorname{ad}\left(E_{G}^{\prime}\right)^{*}$ induced by $\nabla^{\prime}$.) Then there is a unique connection $\nabla^{\prime \prime}$ on the principal $G$-bundle $E_{G}^{\prime}$ such that $\nabla^{\prime}$ is obtained from $\nabla^{\prime \prime}$ by extension of structure group.

Proof. Let $E_{\mathrm{GL}(\mathfrak{g})}^{\prime}$ be the smooth principal GL(g)-bundle over $M^{\prime}$ defined by $\operatorname{ad}\left(E_{G}^{\prime}\right)$. Since $E_{\mathrm{GL}(\mathfrak{g})}^{\prime}$ is obtained by extending the structure group of $E_{G}^{\prime}$ using the adjoint representation of $G$, a connection on $E_{G}^{\prime}$ induces a connection on $E_{\mathrm{GL}(\mathfrak{g})}^{\prime}$. Since the kernel of the adjoint 
representation of $G$ is finite, there can be at most one connection on $E_{G}^{\prime}$ inducing a given connection on $E_{\mathrm{GL}(\mathfrak{g})}^{\prime}$.

Let $\nabla_{0}^{\prime}$ be the smooth one-form on $E_{\mathrm{GL}(\mathfrak{g})}^{\prime}$ defining the connection $\nabla^{\prime}$. Let

$$
\rho: E_{G}^{\prime} \rightarrow E_{\mathrm{GL}(\mathfrak{g})}^{\prime}
$$

be the natural map. We will show that $\rho^{*} \nabla_{0}^{\prime}$ is a connection on $E_{G}^{\prime}$. Let

$$
\rho_{G}: G \rightarrow \mathrm{GL}(\mathfrak{g})
$$

be the adjoint representation of $G$. The kernel of $\rho_{G}$ coincides with the center $Z(G) \subset G$. Hence the image $\rho\left(E_{G}^{\prime}\right)$ is a principal $G / Z(G)$-bundle over $M^{\prime}$. Furthermore, $\rho_{G}(G)$ is a connected component of the subgroup $\operatorname{Aut}(\mathfrak{g}) \subset \mathrm{GL}(\mathfrak{g})$. Therefore, the condition

$$
\left[\nabla_{v}^{\prime}(s), t\right]+\left[s, \nabla_{v}^{\prime}(t)\right]=\nabla_{v}^{\prime}([s, t])
$$

in the lemma means that the pullback $\rho^{*} \nabla_{0}^{\prime}$ on $E_{G}^{\prime}$ is a $\mathfrak{g}$-valued one-form. Consequently, $\rho^{*} \nabla_{0}^{\prime}$ defines a connection on $E_{G}^{\prime}$. It is easy to see that this connection on $E_{G}^{\prime}$ defined by $\rho^{*} \nabla_{0}^{\prime}$ induces the connection $\nabla^{\prime}$ on $E_{\mathrm{GL}(\mathfrak{g})}^{\prime}$.

Continuing with the proof of the proposition, let $\nabla^{G}$ be the connection on $E_{G}$ obtained from the connection $\nabla$ on $\operatorname{ad}\left(E_{G}\right)$ using Lemma 3.3. Since $\nabla$ is flat holomorphic, it follows immediately that the connection $\nabla^{G}$ on $E_{G}$ is also flat holomorphic.

Finally, since $\nabla$ preserves the filtration in (3.3), and $\operatorname{ad}\left(E_{P}\right)=\mathcal{V} \cap \tau\left(\operatorname{ad}\left(E_{G}\right)\right)$, the connection $\nabla^{G}$ on $E_{G}$ induces a connection on $E_{P}$. In other words, the connection $\nabla^{G}$ is the extension of a connection on the $P$-bundle $E_{P}$. This connection on $E_{P}$ clearly has the property stated in the proposition.

\section{REFERENCES}

[AB] B. ANCHOUCHE and I. BISWAS, Einstein-Hermitian connections on polystable principal bundles over a compact Kähler manifold, Amer. J. Math. 123 (2001), 207-228.

[ABi] H. AZAD and I.BISWAS, On holomorphic principal bundles over a compact Riemann surface admitting a flat connection, Math. Ann. 322 (2002), 333-346.

[Bor] A. Borel, Linear algebraic groups. Second edition. Graduate Texts in Mathematics, 126. Springer-Verlag, New York, 1991.

[Bo] R. BotT, Homogeneous vector bundles, Ann. Math. 66 (1957), 203-248.

[DPS] J.-P. Demailly, T. Peternell and M. Schneider, Compact complex manifolds with numerically effective tangent bundles, J. Algebraic Geom. 3 (1994), 295-345.

[Do] S. K. Donaldson, Infinite determinants, stable bundles and curvature, Duke Math. J. 54 (1987), 231-247.

[Mo] C. Mourougane, Images directes de fibrés adjoints, Publ. Res. Inst. Math. Sci. Kyoto Univ. 33 (1997), 893-916.

[Ra] A. Ramanathan, Stable principal bundles on a compact Riemann surface, Math. Ann. 213 (1975), 129_ 152.

[RS] A. RAmANATHAN and S. SUbRAmAnian, Einstein-Hermitian connections on principal bundles and stability, J. Reine Angew. Math. 36 (1984), 269-291.

[Si] C. T. Simpson, Higgs bundles and local systems, Inst. Hautes Études Sci. Publ. Math. 75 (1992), 5-95. 
[UY] K. UHLENBECK and S.-T. YAU, On the existence of Hermitian-Yang-Mills connections in stable vector bundles, Commun. Pure Appl. Math. 39 (1986), 257-293.

SCHOOL OF MATHEMATICS

TATA InSTITUTE OF Fundamental RESEARCH

HOMI BHABHA ROAD

BOMBAY 400005

INDIA

E-mail address: indranil@math.tifr.res.in subramnn@math.tifr.res.in 